\title{
Foods and beverages provided in out of school hours care services: an observational study
}

\author{
Ruth K. Crowe ${ }^{1,2^{*}}$, Yasmine C. Probst ${ }^{1,2}$, Jennifer A. Normann ${ }^{2,3,4}$, Susan E. Furber ${ }^{2,3}$, Rebecca M. Stanley ${ }^{2,4}$, \\ Sarah T. Ryan ${ }^{4}$, Cecilia Vuong ${ }^{5}$, Megan L. Hammersley ${ }^{2,4}$, Karen Wardle ${ }^{5}$, Lisa Franco ${ }^{2,3}$, Michael W. Beets ${ }^{6}$, \\ R. Glenn Weaver ${ }^{6}$, Marc Davis ${ }^{7}$, Christine Innes-Hughes $^{7}$ and Anthony D. Okely ${ }^{2,4}$
}

\begin{abstract}
Introduction: Out of school hours care (OSHC) is a fast-growing childcare setting in Australia, however the types of foods and beverages offered are relatively unknown. This study describes the food and beverages offered and investigates sector-level and setting-level factors which may impact OSHC in meeting the Australian Dietary Guidelines (ADG).

Methods: This cross-sectional, observational study was conducted in 89 OSHC services (between 2018 and 2019). Food and beverages offered, kitchen facilities and menus were captured via direct observation. Foods were categorised into five food groups or discretionary foods, based on the ADG, and frequencies determined. Short interviews with OSHC directors ascertained healthy eating policies, staff training, food quality assessment methods and food budgets. Fisher's exact test explored the influence of sector-level and setting-level factors on food provision behaviours.
\end{abstract}

Results: Discretionary foods $(1.5 \pm 0.68)$ were offered more frequently than vegetables $(0.82 \pm 0.80)(p<.001)$, dairy $(0.97 \pm 0.81)(p=.013)$ and lean meats $(0.22 \pm 0.54)(p<.001)$. OSHC associated with long day care and reported using valid food quality assessment methods offered more lean meats ( $p=.002$, and $p=.004$ ). Larger organisations offered more vegetables $(p=.015)$ and discretionary foods $(p=.007)$. Menus with clearly worded instructions to provide fruits and vegetables daily offered more fruit $(p=.009)$, vegetables $(p<.001)$ and whole grains $(p=.003)$. No other sector or setting-level factors were associated with services aligning with the ADG.

Conclusion: Future interventions could benefit from trialling menu planning training and tools to assist OSHC services in NSW meet the ADG requirements.

Keywords: Healthy eating, Food environment, Nutrition, Dietary guideline, Out of school hours care, Afterschool care, Child care, Primary-school children

\footnotetext{
*Correspondence: rc101@uowmail.edu.au

${ }^{1}$ School of Medicine, Science Medicine and Health, University

of Wollongong, Wollongong, NSW, Australia

Full list of author information is available at the end of the article
}

\begin{abstract}
Introduction
The vast majority of Australian children do not meet the national recommendations for consumption of vegetables $(99.6 \%)$ or lean meats and meat alternatives (99.3\%); and girls under-consume dairy products (96.1\%) [1]. Furthermore, Australian children receive almost $40 \%$ of their energy from discretionary foods and beverages, high in
\end{abstract}


saturated fat, salt or sugars $[1,2]$. Poor dietary intake during childhood is likely to progress into adulthood and is linked to an increased risk of obesity and disease [3, 4]. Research has identified the important role that school and childcare services can play in fostering healthy food environments and promoting healthy eating practices in children [5,6]. A number of interventions have focused on Australian schools and early childhood education and care settings (0-5 years) [6-9]. Less attention, however, has been given to food environments within the out of school hours care (OSHC) setting for primary school aged children (5-12 years). Although, studies conducted within Afterschool programs across the United States of America have frequently reported less than optimal food environments, with many services not achieving healthy eating standards [10-12].

OSHC is the second largest childcare setting in Australia, with children spending an average of 11 hours per week in OSHC [13]. The number of child enrolments have increased substantially from 162,000 in 2002, to 453,850 children in 2018 [13], with New South Wales (NSW) recording the highest proportion of child enrolments (144,140, 32\%) in Australia [13].

While attending OSHC, children are provided with food and beverages (breakfast, morning or afternoon tea) by the OSHC service. Childcare in Australia is governed by the Australian Children's Education and Care Quality Authority to ensure that child education and care settings meet the National Quality Framework and its seven National Quality Standards [14]. However, the only directive regarding the quality of food and beverages is that all food provided should be consistent with the Australian Dietary Guidelines (ADG) and water should always be available. The ADG are whole day guidelines which recommend consumption of a variety of foods from the five food groups (fruit, vegetables, grains, lean meats or alternatives and dairy) and to limit discretionary foods. Providing foods that align with the ADG can assist children to meet their daily nutritional requirements, especially of under-consumed food groups (e.g. vegetables) [15]. However, a part from this, no sector-specific guidelines exist.

Data relating to the types of food and beverages offered in OSHC services is lacking, with the most recent Australian studies conducted prior to $2003[16,17]$ and none have explored potential environmental factors that may be associated with providing healthy food options. As attendance in OSHC is growing [13], it is important to understand what foods and beverages are available for children and what sector and setting-level factors may influence the availability of healthy foods.

A socio-ecological model is a useful framework when exploring food environments as it accounts for the complex connection between sectors (e.g. government policy and legislation), settings (e.g. childcare), and individual factors that impact upon a person's food choices and consumption behaviours [18]. For the purpose of this study, the focus is on connections between the food environment with sectors and settings.

This study aimed to 1) describe the types of foods and beverages offered within OSHC afterschool settings in two local health districts in New South Wales (NSW), Australia, and 2) examine how foods provided by services differed by sector-level and setting-level factors.

\section{Methods \\ Study design and setting}

A cross-sectional observational study was undertaken in OSHC services operating in the afterschool period (15:00 - 18:00) across two local health districts in NSW, Australia. The two districts contain metropolitan, suburban and rural communities and a diverse range of socioeconomic areas $[19,20]$. This study was conducted according to the guidelines of the declaration of Helsinki, and approval was granted by the University of Wollongong Human Research Ethics Committee (HE17/490). The reporting of this research was guided by the Strengthening the Reporting of Observational Studies in Epidemiology (STROBE) checklist [21].

\section{Sample selection and recruitment}

OSHC services from within the two districts were eligible to participate if they: operated from 15:00 - 18:00 during school terms; had a minimum of five primary schoolaged children (5-12 years) enrolled each day; provided at least one afternoon snack; and were not exclusively advertised as a homework or physical activity-related club (e.g. dance academy or football club). Written informed consent was obtained from OSHC directors. OSHC service staff and parents were notified of the study via OSHC internal communication avenues and all information relating to this study was displayed at each OSHC service for a minimum of two weeks prior to data collection and during the data collection period. A detailed methodology has been previously published [22].

\section{Data collection}

All data were collected between March 2018 to April 2019 by trained data collectors. This training included classroom simulation and practical on-site training at a local, non-participating OSHC service. Data were collected from each participating OSHC service on two, non-consecutive weekdays.

\section{Food and beverage Observation and Categorisation}

The types of food and beverages served to children were recorded via direct observation by trained data 
collectors, following previously published protocols $[12$, 23, 24]. Within this study, foods observed were reported as offered, rather than consumed, as the purpose of this study was to describe the types of foods served to children within the OSHC afterschool setting. Prior to foods being offered to children, they were recorded and photographed by a data collector. If foods were made prior to our arrival (e.g. cooked meals or prepared sandwiches) recipes were collected and all available nutritional labels were documented and photographed. Following this, food items were coded into the five food groups of the ADG $[4,25]$ with an additional sixth group for discretionary food items. Food groups were coded dichotomously, as offered or not offered and each food group was sub-categorised. For sub-categories, see Table 1.

\section{Sector-level factors}

The Australian Bureau of Statistics, Socio-Economic Indexes for Areas, was used to classify each service into tertiles as being within a low, medium or high socio-economic area [26].

\section{Setting-level factors}

A brief, semi-structured interview was conducted with each OSHC service director during one of the site visits. The interview explored the service's healthy eating policies and practices and was guided by the Healthy Afterschool Activity and Nutrition Documentation tool [27]. If the service had a healthy eating policy a copy of the policy was requested. Policies were assessed and the level of detail was categorised as: non-specific (limited detail, only states foods will be offered that align with the ADG) [25] or specific (clear objectives e.g. serve a fruit and vegetable each day, beverages will only include water and milk). Practices that were assessed included a) annual staff nutrition training: no training $(<1$ hour per year) or training ( $\geq 1$ hour per year); b) the use of food assessment methods to assess weekly menus against the ADGs: non-valid assessments (no or limited assessment) or valid assessments (dietitian or use of a nutrition calculator); and c) grocery expenditure which was divided by the number of children per day to calculate daily expenditure.

In addition to exploring service's policies and practices, we also observed food preparation facilities and menus. The types of facilities were coded as either: limited (sink, refrigerator, limited bench space and food storage space); moderate (sink, refrigerator, microwave, moderate bench space and food storage space); or complete (sink, refrigerator, microwave, oven, stove, dishwasher, large bench space and food storage space).

Menus were photographed on each observation day. Although no menu collected contained specific instructions or a checklist to offer all five food groups, a portion of menu templates contained instructions or a checklist component to serve fruit, or fruit and vegetables daily. Therefore, for the purpose of this analysis, menus were reviewed regarding their inclusion of fruits and vegetables and were coded as: none (no food groups mentioned), non-specific (serve a fruit or a vegetable daily) and specific (serve a fruit and a vegetable daily).

\section{Data analysis}

Data were summarised using descriptive statistics, including frequency, mean (standard deviation (SD)) and median for food and beverages observed to be offered across two observation days. Shapiro-Wilk tests indicated that food data were skewed. A McNemar test was applied to explore if discretionary foods were offered more frequently than each of the five food groups (vegetables, fruit, grains, lean meats or meat alternatives, and dairy) with the McNemar-Bowker's test of symmetry used to investigate differences between site visits. For categories that showed significance, a chi-square (McNemar test) was used to determine on which days the significant difference occurred. To control for multiple t-tests, a $p$ value of $<0.017$ was applied. Fisher's Exact test was used to explore whether foods offered that aligned with the ADG differed across sector and setting-level factors. An alpha level of $p<0.05$ was used for these analyses. All analyses were conducted using SPSS software (version 24, IBM Corporation, Armonk, NY, USA). Data were analysed in 2019-2020.

\section{Results}

There were a total of 243 OSHC services in the two Local Health Districts at the time of recruitment, a flowchart of the recruitment process and categorisation of service types has been presented (Fig. 1). In total, 89 OSHC services (37\%) participated in the study with 4,408 children in attendance across the two observation days. All services were privately owned and located within school grounds (73\%), community halls (17\%) and early childhood settings (e.g. long day care) (10\%). All services ran between 15:00 and 18:00, on at least four days a week and provided an afternoon snack to children.

\section{Food groups and meal types offered}

Fruit was the most frequently observed food group offered as part of the afternoon snack $(1.82 \pm 0.47)$, followed by discretionary foods $(1.5 \pm 0.68)$, refined grains $(0.98 \pm 0.78)$, dairy $(0.97 \pm 0.81)$, vegetables $(0.82 \pm 0.80)$, whole grain $(0.45 \pm 0.75)$ and lean meats $(0.22 \pm 0.54)$. Findings from the McNemar-Bowker's test indicate that discretionary foods were offered more frequently than 
Table 1 Food and beverages provided by OSHC services over a two day observation period

Food Description

Fruit

Fresh fruit

Dried fruit

Canned fruit

Vegetables

Fresh/raw

Cooked vegetables in meals

\section{Dairy or alternatives}

Cheese

Milk

Light milk

Full cream milk

Dairy alternatives

Yoghurt

Flavoured (full fat)

Flavoured (reduced fat)

\section{Lean meats or alternatives}

Beef or chicken

Chickpeas/hummus/baked beans

Eggs

Tuna

\section{Grains}

High fibre/whole grain

Refined grain

\section{Discretionary foods}

aprocessed meats

bHigh salt/ low fibre snacks

'Sweet snacks

${ }^{d}$ Confectionary

eDiscretionary dairy

Sauces and spreads

Sauces (Tomato / Barbecue / sweet chilli)

Cream cheese

Margarine

\section{Beverages}

Water

100\% fruit juice

Fruit drink

Milo $^{\text {TM }}$ (chocolate drink)
Days (\%) food \& beverages were observed to be offered $(n=176)$

166 (94)

165 (94)

8 (5)

$6(3)$

77 (44)

67 (38)

$10(6)$

$90(51)$

66 (37)

$16(9)$

8 (5)

8 (5)

$3(2)$

15 (9)

5 (3)

$10(6)$

19 (11)

8 (5)

8 (5)

1 (1)

2 (1)

$124(70)$

$41(23)$

88 (50)

142 (81)

48 (27)

50 (28)

32 (18)

52 (29)

$16(9)$

37 (21)

23 (13)

50 (28)

2 (1)

2 (1)

4 (2)

Foods may not have been provided in isolation, but with a combination of other reported items

OSHC out of school hours care

a includes chicken nuggets, sausages, hotdogs/frankfurts, chorizo, luncheon meat, salami, cabanossi

b includes two-minute noodles, chips, savoury biscuits $>1800 \mathrm{~kJ} / 100 \mathrm{~g}$, packet soup mix, savoury pastries

c includes chocolate, cakes, muffins, sweet biscuits/ cookies, jelly, muesli bars, sweet pastries

d includes jam, honey, cinnamon sugar, sprinkles/ hundreds-and-thousands

e includes cream, ice-cream, custard, butter, frozen yogurt 


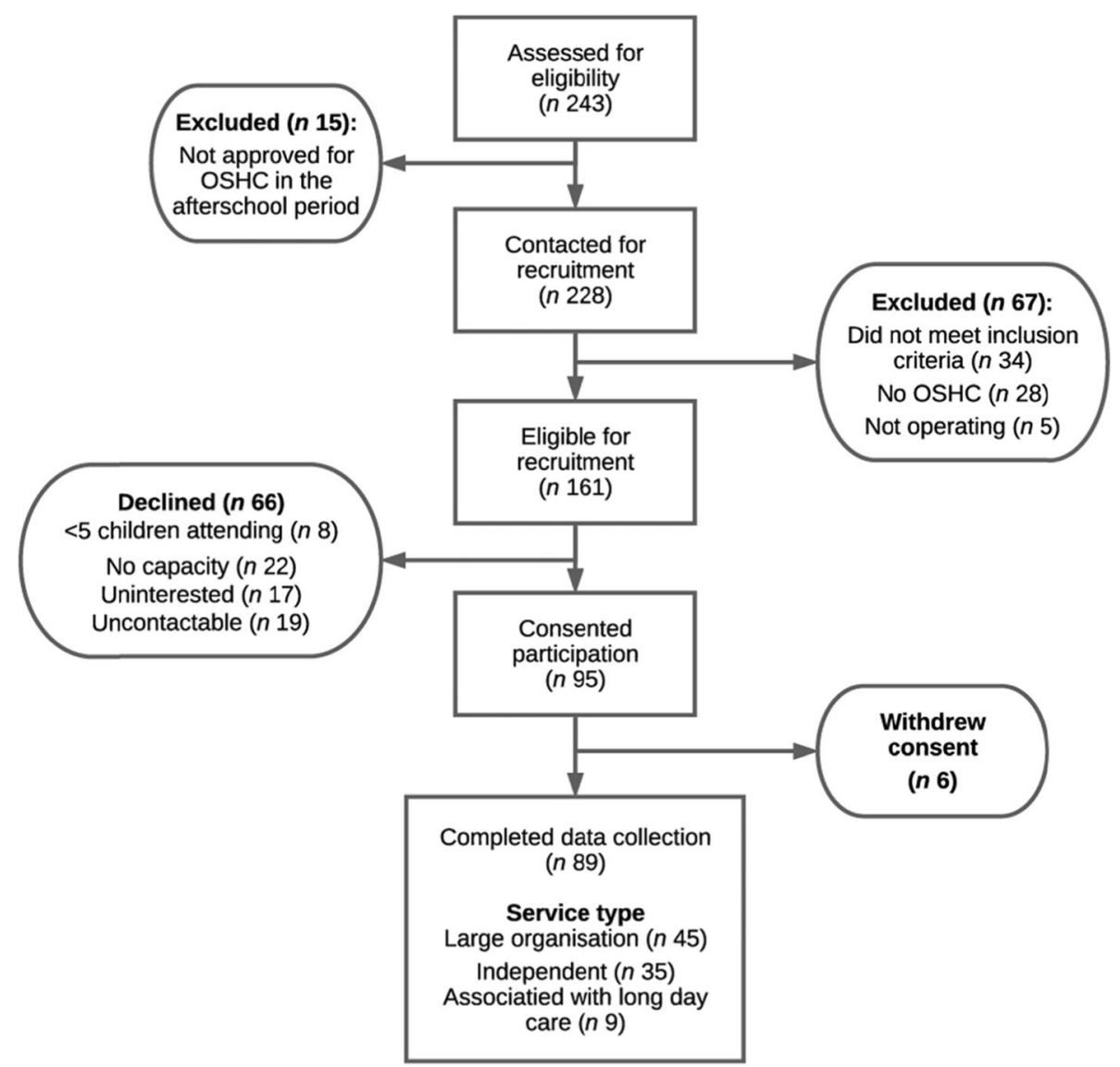

Fig. 1 A flow diagram on recruitment of Out of School Hours Care (OSHC) services

vegetables $(p<0.001)$, dairy $(p=.013)$ and lean meats or their alternatives $(p<0.001)$. The most commonly provided meal types consisted of fruit platters, sandwiches with confectionary fillings, healthy cooked meals and discretionary cooked meals (Table 2).

\section{Sector and setting level factors}

Fifty-seven services (59\%) provided their nutrition policy. Thirty-three services (37\%) were part of a larger organisation and used the policy of the overarching organisation; therefore, 24 unique policies were collected. All policies were very similar, using non-specific language throughout their documentation and, therefore, were excluded from Fisher's Exact Test.

Table 3 presents findings from the Fisher's Exact Test. OSHC services operating out of a long day care facility and those who reported assessing the quality of their menus with valid methods (nutrition calculator or dietitian) offered more lean meats or alternatives $(p=.002$, and $p=.004$ respectfully). OSHC associated with large organisations offered more vegetables $(p=0.015)$ and discretionary foods $(p=0.007)$. OSHC services that had menus which specified serving "fruit AND vegetables" daily, were observed to offer more fruits $(\mathrm{p}=0.009)$, vegetables $(p<0.001)$ and whole grains $(p=0.003)$

\section{Discussion}

This cross-sectional study observed the food and beverages provided to children (5-12 years), across a large sample of OSHC afterschool services. To the authors' knowledge, this is the first observational study to explore both the food and beverages offered and sector-level and setting-level factors that may influence compliance with ADG within Australian OSHC services. We found fruit was the most common food group offered across all observation days, however discretionary foods were observed significantly more than vegetables, dairy and lean meats or their alternatives. Water was the most frequent beverage type offered. Results from Fisher's Exact Test indicate a number of environmental factors were found to be associated with offering food groups aligning with the ADG. 
Table 2 Proportion (\%) of meal type provided to children attending $\mathrm{OSHC}$ in the afterschool period.

\begin{tabular}{lll}
\hline Meal type & Description & $\begin{array}{l}\text { Freq. (\%) } \\
\text { of meals } \\
\text { observed }\end{array}$ \\
\hline Cooked meals & Healthy & \\
& Discretionary & $42(24)$ \\
Sandwiches & $25(14)$ \\
& Confectionary & $47(26)$ \\
Llatter & Sean meat or alternative & $13(7)$ \\
& Fruit & $10(6)$ \\
& Fruit \& Vegetable & $51(29)$ \\
Other & Savoury & $20(11)$ \\
& & $21(12)$ \\
& Dessert & $8(4)$ \\
& Fruit \& Dairy & $19(11)$ \\
\hline
\end{tabular}

Healthy cooked meals include (pasta, rice, curry/ stir-fry)

Discretionary cooked meals include (chicken nuggets, sausage/ hotdog, pasties/ pies/ pizza scroll, two-minute noodles)

Confectionary sandwiches include (fillings of jam/ honey/ sprinkles/ hundreds and thousands)

Meat based sandwiches include (fillings of tuna, chicken breast, eggs)

Savoury platters include (could include a mixture of biscuits, dips, processed meats (cabanossi), cheese or vegetable sticks)

Dessert include (cakes, muffins, slices, sweet biscuits, jelly,

Fruit \& Dairy include (fresh or canned fruits with yogurt or custard)

OSHC out of school hours care

Discretionary foods are recommended to be consumed sometimes and in small amounts [25], yet our results indicate that discretionary foods may frequently be offered by OSHC services. Although the Guidelines are an important national resource, their appropriateness as the sole resource for the OSHC setting may be unsuitable as before-school and after-school services only provide breakfast or an afternoon snack across a child's day. To consider this within the context of a child's day, findings from the National Health Survey reports that discretionary foods, specifically cakes, sweet biscuits and processed meats, are some of the primary sources of energy, saturated fats, added salt and sugars within children's diet [28]. Additionally, a NSW study reported children have on average, 1.5 serves of discretionary foods already within their school lunch boxes $[29,30]$. As Australian children may be exceeding recommended serves of discretionary foods outside of the OSHC setting, it highlights the need for clear healthy eating guidelines specific to the OSHC sector, especially regarding discretionary foods. An example of how clear guidelines and policy within the school setting may have had a positive influence on the $\mathrm{OSCH}$ sector, can be seen by comparing beverage data before and after the introduction of the mandatory cessation of the sale of sugar sweetened beverages in NSW Government schools in 2007 by the NSW Government. Data collected in OSHC services prior to this date reports $24 \%$ of services offering sugary beverages (cordial) to children [17], in comparison to just $1 \%$ of services in the present study.

Other childcare settings, such as Early Childhood Education and Care, have clear sector-specific guidelines ("Caring for Children - birth to 5yrs") [31], to assist their services to offer foods consistent with the ADG [25], Infant Feeding Guidelines [32] and the National Quality Standards [14]. "Caring for Children - birth to 5 years" contains a detailed menu planning section outlining the type and quantity that each food group should provide each day. Serving lean meats or meat alternatives daily is one such recommendation. This recommendation may have indirectly impacted the behaviour of OSHC services associated with long day care (primarily a care setting for 0-5years) and may explain why these services were observed to offer significantly more lean meats within our study; as a portion of the lunch time meal was provided during the afternoon OSHC. Further to this, the use of valid food quality assessment methods (dietitian or nutrition calculator), at least once a year, was also positively associated with services offering more lean meats or their alternative. As lean meats were seldom observed within our sample, the provision of annual menu support, such as dietitian or nutrition calculator, may assist support OSHC services to make achievable improvements to their menu practices and provide foods that align with dietary guideline recommendations.

Although our findings demonstrate OSHC services are regularly providing fruit; vegetables were observed on less than half the observation days. Interestingly, we found significantly more vegetables and, surprisingly, whole grains to be offered at services that used menu planning template with a checklist instructing the provision of "fruit and vegetables" daily. These types of menu planning templates were found mostly in OSHC services associated with large organisations, who disseminated a uniform menu planning template across all of their services and may explain why these services were more likely to offer vegetables. It is however, unclear why whole grains were associated with menu planning templates specifying to offer "fruit and vegetables" and may be a chance finding. Although uniformity in menu templates can equate to positive behaviours such as serving more vegetables, we found that the opposite was also possible. Large organisations in this sample were also linked to offering discretionary foods more frequently, which may be due to organisations within our study using an 
Table 3 Differences in the provision of foods aligning with ADG by sector and setting level factors

\begin{tabular}{|c|c|c|c|c|c|c|c|}
\hline Service Characteristics & $\begin{array}{l}{ }^{\text {a Fruit }} \\
\text { (\%) }\end{array}$ & ${ }^{b}$ Vegetable (\%) & $\begin{array}{l}\text { 'Dairy/ } \\
\text { alternatives } \\
(\%)\end{array}$ & $\begin{array}{l}\text { dLean meats/ } \\
\text { alternatives } \\
(\%)\end{array}$ & $\begin{array}{l}{ }^{\text {e Refined }} \\
\text { Grains (\%) }\end{array}$ & $\begin{array}{l}\text { fWhole } \\
\text { grain (\%) }\end{array}$ & $\begin{array}{l}{ }^{9} \text { Discretionary } \\
\text { (\%) }\end{array}$ \\
\hline \multicolumn{8}{|l|}{ SECTOR -LEVEL } \\
\hline \multicolumn{8}{|l|}{ SEIFA ranking } \\
\hline $\operatorname{Low}(n=40)$ & 95 & 55 & 62 & 15 & 30 & 20 & 85 \\
\hline Medium $(n=31)$ & 96 & 70 & 80 & 20 & 43 & 43 & 97 \\
\hline High $(n=18)$ & 100 & 44 & 50 & 20 & 39 & 28 & 90 \\
\hline \multicolumn{8}{|l|}{ SETTING-LEVEL } \\
\hline \multicolumn{8}{|l|}{ Service Type } \\
\hline Large organisation $(n=43)$ & 100 & $72^{*}$ & 70 & 11 & 49 & 35 & $98^{*}$ \\
\hline Independent $(\mathrm{n}=37)$ & 92 & 40 & 62 & 14 & 27 & 27 & 87 \\
\hline Long day care $(\mathrm{n}=9)$ & 100 & 62 & 62 & $63^{*}$ & 12 & 12 & 62 \\
\hline \multicolumn{8}{|l|}{ Kitchen Facilities } \\
\hline Limited $(n=14)$ & 100 & 57 & 71 & 0 & 100 & 43 & 93 \\
\hline Moderate $(n=21)$ & 100 & 75 & 65 & 15 & 80 & 15 & 85 \\
\hline Complete $(n=54)$ & 94 & 52 & 65 & 22 & 87 & 32 & 91 \\
\hline \multicolumn{8}{|l|}{ Staff Training } \\
\hline No training $(n=59)$ & 98 & 60 & 66 & 17 & 36 & 31 & 88 \\
\hline Training $(n=30)$ & 93 & 53 & 67 & 17 & 37 & 27 & 93 \\
\hline \multicolumn{8}{|l|}{ Daily cost of food AUD\$ } \\
\hline$\leq \$ 0.39(n=34)$ & 94 & 59 & 62 & 12 & 47 & 41 & 94 \\
\hline$\$ 0.40-\$ 0.69(n=34)$ & 100 & 49 & 63 & 15 & 24 & 27 & 85 \\
\hline$\geq \$ 0.70(n=21)$ & 95 & 71 & 76 & 29 & 38 & 14 & 91 \\
\hline \multicolumn{8}{|l|}{ Food Quality Assessment } \\
\hline None $(n=44)$ & 100 & 63 & 60 & 9 & 42 & 33 & 88 \\
\hline Non-valid $(n=36)$ & 94 & 50 & 69 & 17 & 25 & 19 & 89 \\
\hline Valid $(n=9)$ & 89 & 67 & 78 & $56^{*}$ & 56 & 56 & 100 \\
\hline \multicolumn{8}{|l|}{ Menu } \\
\hline None $(n=14)$ & $84^{*}$ & 47 & 53 & 26 & 84 & 21 & 79 \\
\hline $\begin{array}{l}\text { Non-specific } \\
\text { (Fruit OR vegetable) }(n=44)\end{array}$ & 100 & 39 & 63 & 21 & 68 & 16 & 92 \\
\hline $\begin{array}{l}\text { Specific } \\
\text { (Fruit AND vegetable) }(n=31)\end{array}$ & 100 & $87^{*}$ & 77 & 7 & 58 & $52^{*}$ & 93 \\
\hline
\end{tabular}

Socio-Economic Index for Areas (SEIFA)

a Includes all fresh, frozen, canned in natural juice (not syrup). Excludes dried fruit and fruit juices

b Includes vegetables that are fresh, frozen, cooked or canned

c Includes fish, eggs, lean meat and poultry, nuts, seeds, legumes and beans

d Includes milk, cheese, yoghurt, milk alternatives (calcium fortified alternatives). Excludes cream, sour cream, dairy desserts or iced confectionary (ice cream or frozen yoghurts)

e Includes all grains, bread, cereals, rice, pasta, noodles, couscous and polenta

${ }^{f}$ Includes all grains products specified as whole grain, whole meal, rye, barley, oats and quinoa.

${ }^{g}$ Includes cream, sweet biscuits, cakes, pastries, pies, processed meat, chips or savoury crackers $>1800 \mathrm{~kJ} / 100 \mathrm{~g}$, high sugar/ salt/ fat spreads, sugar-sweetened beverages and lollies/ candy.

ADG Australian Dietary Guidelines

* Indicates values are significant $p<0.05$

identical daily menu across each of their services, with their daily menu consisting of sandwiches; processed meat, cream cheese, jam and honey with a fruit and vegetable platter. Producing menus compliant with dietary guidelines has been identified throughout the literature as a complex task [33] with a number of key barriers including, a lack of training, resources and ongoing support [34-36].

Evidence from systematic literature reviews indicates that in order to make significant behaviour 
changes, and to support menu development to align with dietary guidelines, multi-component interventions are needed within childcare settings [5, 35]. A randomised controlled trial, conducted in NSW, within the early childhood education and care sector applied a multicomponent intervention focusing on: staff training (menu planning workshop), menu audits and feedback, face-to-face support and additional resources (menu planning template/ checklist) [37]. The intervention found a significant improvement in recommended food groups on planned menus compared to the control group, and a significant increase in child vegetable and fruit consumption was evident in the intervention group [37]. Although nutrition training was not found to be associated with food groups served within our study, this may be due to the type, quality and frequency of the training provided. Currently in NSW, there is no tailored nutrition training, menu development support or feedback available to OSHC services and therefore any training provided to OSHC staff would have been organised internally and may not have been sufficient to produce behaviour change. Future interventions should trial the effect of sector-specific guidelines, nutrition training and menu planning tools to support effective behaviour changes within the OSHC setting.

The findings in our study need to be considered in context of its limitations. Firstly, although this study sample included a number of services from a diverse geographical landscape, all services were recruited from within two local health districts in NSW and may not be representative across NSW or Australia. Secondly, this study observed food groups provided by OSHC and did not report on the number of servings per child nor actual consumption of food and beverages. Finally, there is potential that some self-reported data may have been misreported, as desirable practices rather than actual practices.

\section{Conclusion}

Findings from this study indicate that OSHC services in NSW may not be providing foods in accordance with ADG, specifically for vegetables, lean meats and their alternatives, dairy and discretionary foods. Introducing a menu planning tool specific to the OSHC setting may be a useful and cost-effective resource to provide a variety of food groups aligning with the dietary guidelines. Future research should focus on the impact of multiple-component interventions (such as the development of sector-specific guidelines, training opportunities, and menu planning tool) on the quality of foods offered within NSW OSHC services.

\section{Abbreviations}

ADG: Australian Dietary Guidelines; OSHC: Out of school hours care; NSW: New South Wales.

\section{Acknowledgements}

None.

\section{Financial disclosure}

No Financial disclosures were reported by the authors of this paper.

\section{Authors' contributions}

Ruth Crowe is a PhD candidate of this study, she has worked with the research team to develop the study design and methodology, led data collection, training, conducted data analysis, interpretation and write up of this manuscript. Yasmine Probst and Rebecca Stanley are PhD supervisors on this project. They have contributed to the study design, interpretation of the data, and provided support through the development and revised the manuscript. Jennifer Norman, Susan Furber, Lisa Franco, Megan Hammersley, Cecilia Vuong, Karen Wardle, Christine Innes-Hughes, and Marc Davis are a part of the Prevention Research Support Program working committee, offering valuable insight and context of local health districts. Jennifer Norman, Megan Hammersley and Susan Furber have made significant editorial contributions to this manuscript. Sarah Ryan is the project manager who has provided administrative support to assist the progression of this project. Michael Beets and Glenn Weaver have contributed to the study design, methodology and training in data collection protocols. Professor Anthony Okely is the chief investigator of this study, contributing to the study design, methodologies, interpretation of the data and is a PhD supervisor on this project. All co-authors have read and approved the final manuscript. This manuscript has not been submitted or published in any other journal.

\section{Funding}

This research has been conducted with the support of the Australian Government Research Training Program Scholarship. This work was supported by the Prevention Research Support Program, funded by the New South Wales Ministry of Health. We declare the funding body has had no influence on the study design, data collection, analysis, interpretations of the findings or writing of this manuscript.

\section{Availability of data and materials}

The datasets generated and analysed during the current study are not publicly available but are available from the corresponding author on reasonable request.

\section{Declarations}

Ethics approval and consent to participate

This study was conducted according to the guidelines of the declaration of Helsinki, and approval was granted by the University of Wollongong Human Research Ethics Committee (HE17/490). Written informed consent was obtained from each OSHC director. Due to the observational nature of this research, methods were determined as low risk and a passive consent was applied to this study.

\section{Consent for publication \\ Not applicable}

\section{Competing interests}

The authors have declared there is no competing interests

\section{Author details}

${ }^{1}$ School of Medicine, Science Medicine and Health, University of Wollongong, Wollongong, NSW, Australia. ${ }^{2}$ Illawarra Health and Medical Research Institute, University of Wollongong, Wollongong, NSW, Australia. ${ }^{3}$ Health Promotion Service, Illawarra Shoalhaven Local Health District, Warrawong, NSW, Australia. 
${ }^{4}$ Early Start, School of Health and Society, University of Wollongong, Wollongong, NSW, Australia. ${ }^{5}$ Health Promotion Service, South Western Sydney Local Health District, Liverpool, NSW, Australia. ${ }^{6}$ Exercise Science, Arnold School of Public Health University of South Carolina, Columbia, SC, USA. ${ }^{7}$ Centre for Population Health, St Leonards, NSW, Australia.

Received: 1 September 2021 Accepted: 25 January 2022 Published online: 11 February 2022

\section{References}

1. Australian Bureau of Statistics. Australian Health Survey: Consumption of food groups from the Australian Dietary Guidelines; 2016. p. 8-92. Available from: https://www.ausstats.abs.gov.au/ausstats/subscriber.nsf/0/77E3E65E 4D967249CA2581F40016ABD6/\$File/4364.0.55.012 - consumption of food groups from australian dietary guidelines.pdf. Accessed 11 Feb 2021.

2. Australian Institute of Health and Welfare. Nutrition across the life stages; 2018Available from: https://www.aihw.gov.au/getmedia/fc5ad42e-08f5-4f9a9ca4-723cacaa510d/aihw-phe-227.pdf.aspx?inline=true. Accessed 4 Aug 2020.

3. Craigie AM, Lake AA, Kelly SA, Adamson AJ, Mathers JC. Tracking of obesity-related behaviours from childhood to adulthood: A systematic review. Maturitas. 2011; Nov70(3):266-84.

4. National Health and Medical Research Council. Australian Dietary Guidelines. 2013. Available from: www.nhmrc.gov.au/guidelines-publications/ n55. Accessed 8 Dec 2020

5. Mikkelsen MV, Husby S, Skov LR, Ja Perez-Cueto F. A systematic review of types of healthy eating interventions in preschools. Nutri J. 2014;13(56).

6. Bennett L, Burns S. Implementing health-promoting schools to prevent obesity. Health Educ. 2020;120(2):197-216.

7. Colin Bell A, Davies L, Finch M, Wolfenden L, Francis J L, Sutherland R, et al. An implementation intervention to encourage healthy eating in centre-based child-care services: Impact of the Good for Kids Good for Life programme. Public Health Nutr. 2015;18(9):1610-9.

8. Wolfenden L, Nathan N, Janssen LM, Wiggers J, Reilly K, Delaney T, et al. Multi-strategic intervention to enhance implementation of healthy canteen policy: A randomised controlled trial. Implement Sci. 2017;12(1):111. https://doi.org/10.1186/s13012-016-0537-9.

9. Wolfenden L, Barnes C, Jones J, Finch M, Wyse RJ, Kingsland M, et al. Strategies to improve the implementation of healthy eating, physical activity and obesity prevention policies, practices or programmes within childcare services. Cochrane Database Syst Rev. 2020;2020(2):1-25.

10. Beets MW, Weaver RG, Tilley F, Turner-Mcgrievy G, Huberty J, Ward DS, et al. Salty or sweet? Nutritional quality, consumption, and cost of snacks served in afterschool programs. J Sch Health. 2015 Feb 1;85(2):118-24.

11. Beets MW, Weaver RG, Turner-McGrievy G, Huberty J, Moore JB, Ward DS, et al. Two-Year Healthy Eating Outcomes: An RCT in Afterschool Programs. Am J Prev Med. 2017 Sep;53(3):316-26.

12. Beets MW, Weaver RG, Turner-McGrievy G, Huberty J, Ward DS, Freedman D, et al. Making Healthy Eating Policy Practice: A Group Randomized Controlled Trial on Changes in Snack Quality, Costs, and Consumption in After-School Programs. Am J Heal Promot. 2016;30(7):521-31.

13. Australian Government Department of Education and Training. Early Childhood and Child Care in Summary. 2018. Available from: https://docs. education.gov.au/system/files/doc/other/eccc_in_summary_jun_quart er_2018_0.pdf. Accessed 23 April 2021.

14. Australian Children's Education and Care Quality Authority. Guide to the National Quality Framework. 2018. Available from: https://www.acecqa.gov.au/ sites/default/files/2020-01/Guide-to-the-NQF_2.pdf. Accessed 19 Dec 2019.

15. Marangoni F, Martini D, Scaglioni S, Sculati M, Donini LM, Leonardi F, et al. Snacking in nutrition and health. Int J Food Sci Nutr. 2019;70(8):909-23.

16. Sangster,.J, Cooke, L., Eccleston P. "What's to eat?" Nutrition and food Safety needs in out-of-school hours care. Nutr Diet. 2004;61(3):172-6.

17. Thompson E, Cooper C, Flanagan C, Crawford D, Worsley A. Food and activity in out of school hours care in Victoria. Nutr Diet. 2006 Mar;63(1):21-7.

18. Story M, Kaphingst KM, Robinson-O'brien R, Glanz K. Creating Healthy Food and Eating Environments: Policy and Environmental Approaches. Rev Adv Annu Rev Public Heal. 2008;29:253-72.

19. NSW Government. Illawarra Shoalhaven - Local health districts. Available from: https://www.health.nsw.gov.au/lhd/Pages/islhd.aspx. Accessed 26 Oct 2020.
20. NSW Government. Live, work, care locally South Western Sydney, Local Health District. 2017.

21. Lachat C, Hawwash D, Ocké MC, Berg C, Forsum E, Hörnell A, et al. Strengthening the Reporting of Observational Studies in EpidemiologyNutritional Epidemiology (STROBE-nut): An Extension of the STROBE Statement. PLOS Med. 2016;7(6):e1002036

22. Crowe R, Probst Y, Norman J, Furber S, Franco L, Stanley RM, et al. Healthy eating and physical activity environments in out-of-school hours care: an observational study protocol. BMJ Open. 2020;10:36397.

23. Mozaffarian RS, Wiecha JL, Roth BA, Nelson TF, Lee RM, Gortmaker SL. Impact of an organizational intervention designed to improve snack and beverage quality in YMCA after-school programs. Am J Public Health. 2010;100(5):925-32

24. Weaver RG, Beets MW, Hutto B, Saunders RP, Moore JB, Turner-McGrievy $\mathrm{G}$, et al. Making healthy eating and physical activity policy practice: process evaluation of a group randomized controlled intervention in afterschool programs. Health Educ Res. 2015 Dec;30(6):849-65.

25. National Health and Medical Research Council. Eat for Health Educator Guide. 2013. Available from: www.nhmrc.gov.au/guidelines-publications/ n55b. Accessed 18 Dec 2019.

26. Australian Bureau Of Statistics. 2033.0.55.001 - Census of Population and Housing: Socio-Economic Indexes for Areas (SEIFA), Australia, 2016. Australian Bureau Of Statistics. 2018 Available from: https://www.abs. gov.au/ausstats/abs@.nsf/Lookup/bySubject/2033.0.55.001 2016 Main Features IRSD 19. Accessed 15 Jul 2020.

27. Ajja R, Beets MW, Huberty J, Kaczynski AT, Ward DS. The Healthy Afterschool Activity and nutrition documentation instrument. Am J Prev Med. 2012 Sep;43(3):263-71.

28. Johnson B, Bell L, Zarnowiecki D, Rangan A, Golley R. Contribution of Discretionary Foods and Drinks to Australian Children's Intake of Energy, Saturated Fat, Added Sugars and Salt. Children. 2017;4(12):104.

29. Brennan L, Miles CL, Mitchell S, Matthews J. Changes in the content of children's school lunches across the school week. Heal Promot J Aust. 2010;21(3):196-201.

30. Sutherland R, Nathan N, Brown A, Yoong S, Reynolds R, Walton A, et al. A cross-sectional study to determine the energy density and nutritional quality of primary-school children's lunchboxes. Public Health Nutr. 2020;23(6):1108-16. https://doi.org/10.1017/S1368980019003379.

31. NSW Ministry of Health. Caring for Children Birth to 5 years. 2014. Available from: www.healthykids.nsw.gov.au. Accessed 29 Jul 2020.1-180 p

32. NHMRC. Infant feeding guidelines - Information for health workers. 2012 Available from: http://www.nhmrc.gov.au. Accessed 8 Oct 2020.

33. Grady A, Stacey F, Seward K, Finch M, Jones J, Yoong SL. Menu planning practices in early childhood education and care - factors associated with menu compliance with sector dietary guidelines. Heal Promot J Aust. 2020;31(2):216-23.

34. Finch M, Seward K, Wedesweiler T, Stacey F, Grady A, Jones J, et al. Challenges of Increasing Childcare Center Compliance With Nutrition Guidelines: A Randomized Controlled Trial of an Intervention Providing Training, Written Menu Feedback, and Printed Resources. Am J Heal Promot. 2019:33(3):399-411.

35. Seward K, Finch M, Yoong SL, Wyse R, Jones J, Grady A, et al. Factors that influence the implementation of dietary guidelines regarding food provision in centre based childcare services: A systematic review. Vol. 105, Preventive Medicine. Academic Press Inc.; 2017. p. 197-205.

36. Grady A, Wolfenden L, Wiggers J, Rissel C, Finch M, Flood V, et al. Effectiveness of a web-based menu-planning intervention to improve childcare service compliance with dietary guidelines: Randomized controlled trial. J Med Internet Res. 2020;22(2):1-16.

37. Seward K, Wolfenden L, Finch M, Wiggers J, Wyse R, Jones J, et al. Improving the implementation of nutrition guidelines in childcare centres improves child dietary intake: Findings of a randomised trial of an implementation intervention. Public Health Nutr. 2018;21(3):607-17.

\section{Publisher's Note}

Springer Nature remains neutral with regard to jurisdictional claims in published maps and institutional affiliations. 